\section{Lens Cleaning - Best Practices Review}

\section{C.V. Duke \\ colin.duke@dial.pipex.com}

There is no substitute for good microscope care. A unit that is stored and covered, with its lens protected in a constant temperature/ humidity environment, which is dust free (in the laboratory away from acid wash areas) speaks for itself. During cleaning, oculars in particular, microscope tubes should be covered to prevent entry of dust. A lens should be inspected with a view to cleaning if there is a lack of contrast, cloudiness or poor definition in the images it produces.

While extolling the virtue of cleaning microscope components, it should be emphasized that cleaning can cause considerable damage if it is done incorrectly. There are three main ways that lenses can be damaged during cleaning.

1. Grinding microscopic grit into soft lens glass and causing scratches.

2. Using caustic agents that remove lens anti-reflection coatings.

3. Applying too much solvent to the glass surface such that the solvent flows around the edge of the lens and attacks the cement between the lens components causing the lens to delaminate.

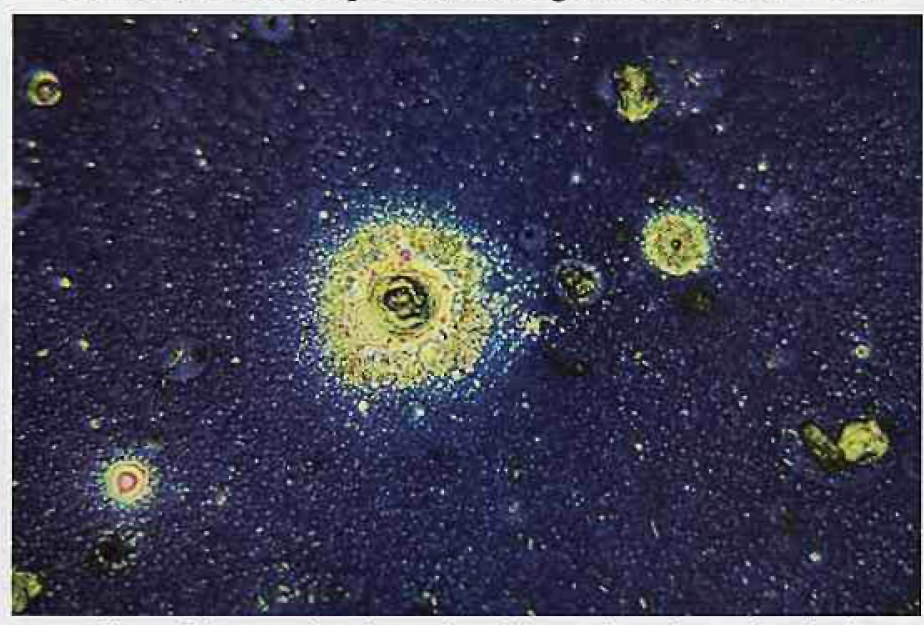

Fig 1: Illustrates close inspection of the top lens of an anti-reflection coated eyepiece that has been removed and placed under a microscope set up for vertical illumination brightfield, showing dust and particulate oils on its surface $x 160$. Images in some cases are a mix of real objects and optical artefacts such as interference fringes, also known as Newton's rings.

\section{Materials: Dusters, Lens Tissue, Papers, \& Brushes}

Mindful of points 1-3, a systematic approach to lens cleaning should be adopted. Dust is the most common contaminant. Begin by blowing off any dust with a dry "Air Duster." Caution should be used to make sure that the product chosen will not disperse aerosol propellants or fluids on to the lens surface, which can occur when the duster is tilted or spraying is prolonged. Specialist high grade air dusters are available for use with optical equipment that feature specialized nozzles and aerosol gas filters. Many prefer the use of a bellows ball or vacuum suction. The trouble with brushes of any sort is that they tend to accumulate grease that can be subsequently transferred to the lens, but they are favoured by many amateurs. Use care.

Lint-free tissues, e.g. Kim Wipes, ${ }^{\text {tw }}$ and particles $x 600$. similar products, are often used for general cleaning of stages and stands-they should not be used to clean lens as they can leave particulate material behind. Kodak manufactures lens cleaning paper and stipulates that it is lintless, safe to use on coated lens, filters, and other glass surfaces. SPI \& Ross manufacture cleaning tissues for critical surfaces. Hard lens papers should be avoided as it does not readily absorb water or oil quickly enough. Natural cotton swabs are not recommended as they can retain small silica particles from the soil and for that reason synthetic swabs, such as Q-tips" are preferred. Little scientific scrutiny has been applied to fibre deposition from such products, but crude evaluation can be made by "rubbing" a slide for each new batch of material as part of a quality control regime.

The use of suitable gloves when handling microscopes is recommended to minimize contamination from fingerprints, body oils, and grease. Specifically designed, lint-free and powder-free gloves can be purchased that are made from a variety of materials (polyethylene to nylon). Finger "Cots" can be purchased (or made) that cover finger tips only. However latex gloves, coated with talc or powder, are not to be worn when using a microscope.

\section{Methods \& Techniques}

There are many variations on technique depending on circumstance - often a combination of approaches may achieve best results. Three basic principles apply: 1 . remove particulates and grit, 2 . dissolve oily deposits in minimal solvent, and 3 . remove contamination by absorbing and or moving residue away via progressive changes of cleaning media. Avoid cleaning lens with abrasive or lint cloths. Never dry-wipe a lens as this may cause scratching. Begin by blowing off dust or loose material with a pressurized optical duster or bellows ball. Flooding or placing solvents directly on the lens is discouraged. Spraying directly onto a lens is highly questionable. Dust areas of the condenser lens prior to objectives in view of minute glass fragments that may be present from broken or chipped slides. Careful fogging of the lens with breath can assist in removing water-soluble residue, although caution should be exercised in regard to excluding saliva. Keep the lens upright during cleaning to allow the tissue to touch the glass surface of the lens only, so solvent does not run into cements or other substances holding the lens in place.

\section{Alternative Techniques}

a. Remove the objective and place it on a flat surface with the front lens uppermost. Fold a piece of lens tissue four or five times to form a narrow triangular shape with a finely pointed end. Moisten the pointed end of the lens tissue with a small amount of cleaner, keeping fingers away from the solvent to avoid contamination by oils. Do not use excessive solvent such that the tissue is saturated. Place the tissue against the objective front lens and begin wiping
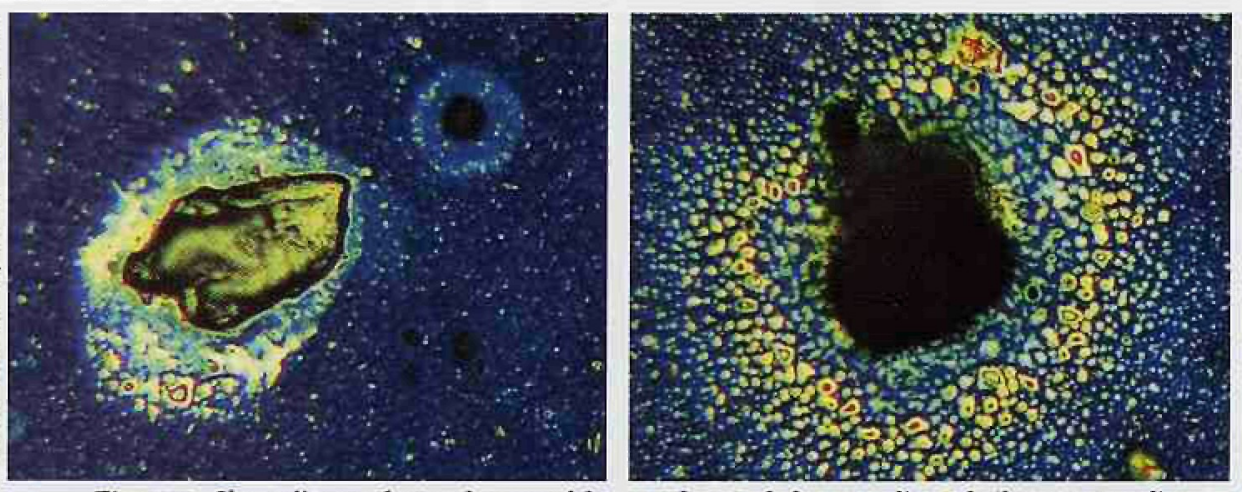

Figs 2-3. Show dirt specks on the coated lens surface and the spreading of oil to surrounding 


\section{Cutting edge science driving the discovery of new medicine.}

$\mathrm{D}$ riving the success of GlaxoSmithKline - the world's leading pharmaceutical organization - is a continual search for innovation. Apart from a research and development capability that sets the benchmark for our industry, we're committed to recruiting and retaining the best and brightest by providing unequaled individual and career development opportunities within our organization. Our mission is clear - to prevent, treat and cure diseases, allowing people around the world to do more, feel better and live longer. We currently have/ opportunities available in our state-of-the-art facility, located in Research Triangłe Park, NC.

\section{Manager of Ultrastructural Pathology}

Using your technical and intellectual expertise, you will lead the Ultrastructural Pathology Laboratory in producing ultrastructural data. This includes producing high-quality interpretive ultrastructural reports for regulatory submissions; implementing SOP's and ensuring that the laboratory is GLP compliant; collaborating with pathologists, study directors, and project representatives to design ultrastructural pathology components of regulatory and investigative studies; and developing and validating new ultrastructural and morphometric laboratory techniques. You'll also provide consultation and support to other scientific staff, serve as a Project Representative on GSK Project Teams for drug development, and design, conduct and interpret non-clinical toxicity studies.

To qualify, you must have a DVM or PhD, at least 5 years experience in ultrastructural data interpretation, recognition as an expert in transmission electron microscopy (TEM), and extensive experience in a GLP environment, preferably in the pharmaceutical industry. Excellent leadership, project management, decisionmaking, problem-solving, presentation and oral/written communication skills are essential, as are demonstrated technical knowledge in conducting toxicity studies with laboratory animals and a strong understanding of regulatory guidelines, GLP regulations, mechanistic animal models, and animal welfare legislation.

(Req. ID: 16193)

\section{Electron Microscopy Specialist}

In this challenging role, you will fix, process, and examine tissues using transmission electron microcopy techniques in a GLP environment. This includes troubleshooting and correcting technical problems; maintaining the TEM laboratory; analyzing results and taking appropriate next steps; using various image analysis systems to measure and quantify data; ensuring that SOP's (Standard Operating Procedures) and GLP's are followed in all laboratory functions; and researching and introducing new techniques to improve processes.

To qualify, you must have at least 4 years of practical experience in Transmission Electron Microscopy (TEM), including a background in a GLP environment, preferably in the pharmaceutical industry. Strong theoretical scientific knowledge, good technical skilss and a working knowledge of laboratory procedures are essential, as are knowledge of GLP practices and experience developing and validating TEM techniques, including immuno-electron microscopy procedures. Background in histotechnology, particularly immunohistochemistry, is a definite asset. (Req. ID: 16194)

GlaxoSmithKline is dedicated to supporting you with career-long opportunities and learning. We offer a competitive benefits and compensation package designed to attract and retain the very best. For confidential consideration and efficient processing of your resume, please visit our website at www.gsk.com. Indicating Reg ID is essential to search. Principals only, no agencies, please.

GSK is proud to promote an open culture, encouraging people to be themselves and giving their ideas a chance to flourish. GSK is an equal opportunity employer.

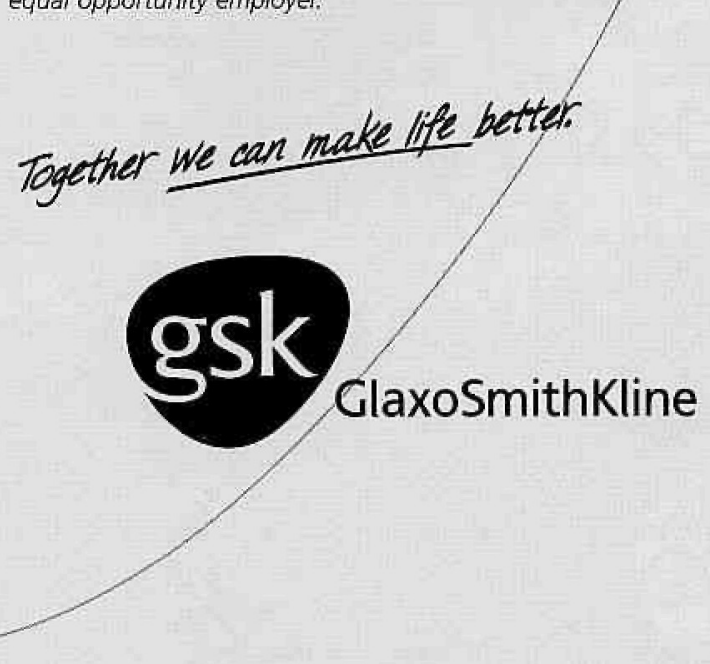


and rotating the lens in a corkscrew motion so that the tissue traverses from the centre of the lens to the outside edge without going back and forth over the same area. In this way the lens can be cleaned up to its edge. Further pressure will cause the tissue to spread sufficiently to clean the whole surface. Remove any obstinate marks by repeating the process. A new and clean piece of tissue should be used when it is necessary to go over the same area on the lens more than once.

b. Roll up some good quality lens tissue, tearing across the roll to leave a brush-like end. This can be dampened with solvent, and then gently applied to soften deposits. Use lens tissue to sweep once only across the lens. Use a different piece of lens paper for each subsequent sweep, if necessary. Monitor the cleaning process using a hand lens or stereomicroscope using light reflection as a progress indicator. Never wipe the lens using a circular motion.

c. The abrasive nature of cotton has led some microscopists to prefer the use of swabs with urethane foam heads (buds). If this method is used, a small amount of xylene is placed on one end of the bud and then gently worked in a spiral fashion and allowed to evaporate. The procedure is repeated with a fresh, clean bud as necessary. Slowly turning the lens and applying gentle pressure in the centre and working outward-this pulls the dirt off the lens instead of redistributing it on the surface. Tipped applicators have advantages over lens paper. They work better on small, concave, or recessed lenses, they are not touched by fingers, and so do not transfer skin oils.

\section{Cleaning OII Immersion (O.I) Lens}

Some have questioned the validity of cleaning O.I. lenses. It would seem intuitive that leaving a lens coated with oil will attract particulate matter and impair image quality. Oil immersion lens are generally wiped clean after use to remove oil from their outer surfaces. If the oil is allowed to dry, the lens will need cleaning with a solvent. Oil left on a lens surface may act in a similar manner to alcohol should capillary action or gravity allow it to travel into the lens cement, causing possible delamination of the lens. For cleaning lenses immersed in Cargille oils, you can use small amounts of carbon tetrachloride, ethyl ether, freon $\mathrm{ff}$, heptane, methylene chloride, naptha, toluene, turpentine, or xylene. Do not use water, alcohol or acetone, as the oil is insoluble to these solvents. In any case, the first time you clean a lens, consult the MSDS (Material Safety Data Sheets) information that is supplied with the immersion oil to see what solvents are recommended. Lens companies tend to recommend the use of one brand of oil for their lenses. Mixing different oil brands or formulations on a lens surface, especially if the lens is not cleaned between oil applications, is not recommended and may promote crystal formation on the lens-leading to severe cleaning issues.

High-grade synthetic immersion oils should be resistant to oxidation as they contain no volatile materials, and therefore should in theory not form gummy deposits. Natural oils, such as Red Cedar oil, may contain volatile components, and are likely to be subject to oxidative degradation, and can often leave gummy residues if not thoroughly removed from the lens.

\section{Cleaning Older Lens}

Caution is required when dealing with older microscopes. Too much solvent applied to lens surfaces may affect the integrity of the mounting cement or, in particular, balsam. With modern microscopes this is less of a problem, due to the use of Polyacrylamates and other more solvent-resistant cements. Old lenses assembled with balsam will delaminate in most aromatic solvents and their use should be avoided. Best cleaning practice in this case is to use a minimal application of a high vapour pressure solvent on a synthetic bud. In very rare cases some antique collectible microscopes had lens coatings that were actually water-soluble.

\section{Fungus \& Mould}

Usually a problem in climates with high humidity. Fungus and mould can cause physical etching of the lens glass due to the possible production of acids and enzymes. Proprietary antifungals can be employed to coat lens used in these environments with consultation of the lens manufacturer.

\section{Anti-Reflection Coatings}

Antireflection coating technology helps to reduce unwanted reflections (flare and ghosts) that occur when light passes through a lens system, ensuring high-contrast images. The microscopist should be aware that caustic substances may damage such layers. Typical coatings contain magnesiun fluoride, therefore, the choice of a commercially available lens cleaner should be based on manufacturer's recommendation and fitness for purpose. There have been reports that the acidic version of Windex may be corrosive to lanthanide oxide and other coatings on glass and to some metal parts - the ammonia version being preferred.

\section{Solvents \& Commercial Cleaners}

It must be emphasised that when cleaning microscope objectives, the manufactures recommended guidelines must be followed, this is particularly important in regard to solvent contact. As a rule, high vapour pressure solvents are used in order to limit contact time with surfaces and cements.

Distilled Water has been recommended for removal of dirt, this may not be wholly effective for a lens that has oily or greasy particulates or smears. Xylene is a popular solvent for lens cleaning and is preferred, particularly for stubborn oily stains. Xylene usually leaves a small residue where the last of it evaporates, which may be removed with a dry swab. Xylene and toluene are toxic carcinogens, and should be handled carefully in a ventilated area. Ethanol, methanol, and isopropyl alcohol may be used to clean lens. Acetone is discouraged on some brands (American Optical, for example) as it will dissolve the paint on the lens mount, which probably will wind up on the lens. Benzine is strongly suspected of causing liver damage and leukaemia; do not use it. Glass distilled reagent grade solvents are preferable.

Some of the larger manufacturers (Leica, Zeiss, Kodak) produce proprietary lens cleaning solutions especially designed for anti-reflective, multi-layer coatings. Fisher brand Optical Lens Cleaner is used for removing oil from objectives or any optical surface and they claim that their cleaner will not affect coated optics or soften the mounting that holds the objective in place. This Optical Lens Cleaner is laboratory safe and non-flammable. Other commercial formulations are available: $R O R^{m}$ (Residial Oil Remover), Windex or Sparkle $e^{\text {tu }}$ are used widely. The use of a dedicated optical brand cleaner for critical lens surfaces is strongly preferred. Always clarify solvent cleaning regimes with the lens manufacturer.

\section{Acknowledgements}

The author acknowledges Microgaphia for the use of micrographs presented in this article (http:/www.micrographia.com).

\section{Disclaimer:}

No responsibility can be accepted for loss or damage resulting from the use of materials or techniques discussed. If in doubt the manufacture must be contacted in the first instance regarding specific questions or the service of a professional service technician sought. 